\title{
Hashimoto's thyroiditis predicts outcome in intrathyroidal papillary thyroid cancer
}

\author{
Vincenzo Marotta', Concetta Sciammarella², Maria Grazia Chiofalo³, \\ Claudio Gambardella4, Claudio Bellevicine5, Marica Grasso6, Giovanni Conzo4, \\ Giovanni Docimo4, Gerardo Botti7, Simona Losito7, Giancarlo Troncone5, \\ Maurizio De Palma8, Laura Giacomelli9, Luciano Pezzullo3, Annamaria Colao' and \\ Antongiulio Faggiano 3

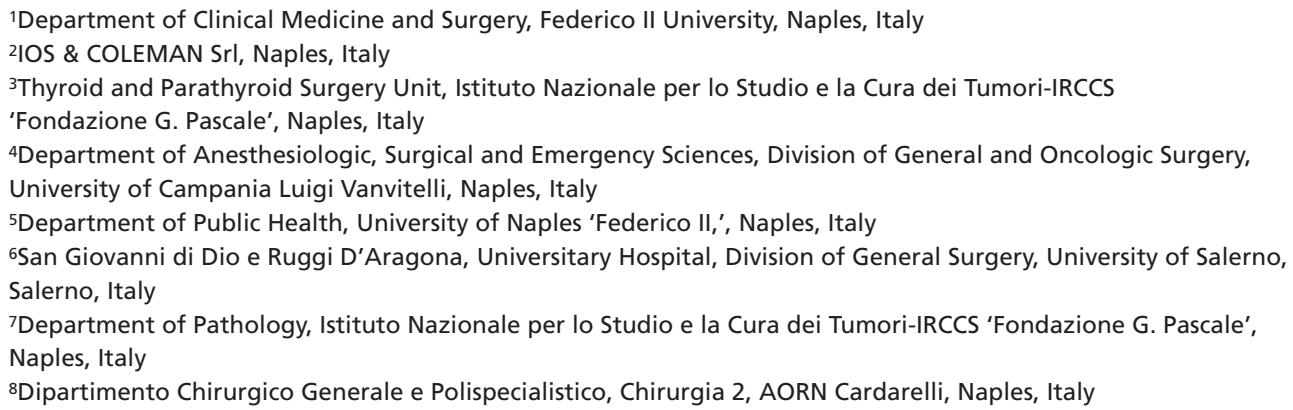

Correspondence should be addressed to V Marotta Email vinc.endo@libero.it

\begin{abstract}
Hashimoto's thyroiditis (HT) seems to have favourable prognostic impact on papillary thyroid cancer (PTC), but data were obtained analysing all disease stages. Given that HT-related microenvironment involves solely the thyroid, we aimed to assess the relationship between $\mathrm{HT}$, as detected through pathological assessment, and outcome in intrathyroidal PTC. This was a multicentre, retrospective, observational study including 301 PTC with no evidence of extrathyroidal disease. Primary study endpoint was the rate of clinical remission. Auxiliary endpoint was recurrence-free survival (RFS). HT was detected in $42.5 \%$ of the cohort and was associated to female gender, smaller tumour size, lower rate of aggressive PTC variants and less frequent post-surgery radio-iodine administration. HT showed relationship with significantly higher rate of clinical remission $(P<0.001, \mathrm{OR}$ 4, 95\% Cl 1.78-8.94). PTCs with concomitant HT had significantly longer RFS, as compared with non-HT tumours $(P=0.004)$. After adjustment for other parameters affecting disease outcome at univariate analysis (age at diagnosis, histology, tumour size and multifocality), prognostic effect of $\mathrm{HT}$ remained significant $(P=0.006$, OR $3.28,95 \% \mathrm{Cl} 1.39-7.72)$. To verify whether HT could optimise the identification of PTCs with unfavourable outcome, we assessed the accuracy of 'non-HT status' as negative prognostic marker, demonstrating poor capability of identifying patients not maintaining clinical remission until final follow-up (probability of no clinical remission in PTCs without HT: $21.05 \%, 95 \% \mathrm{Cl} 15.20-27.93$ ). In conclusion, our data show that HT represents an independent prognostic parameter in intrathyroidal PTC, but cannot improve prognostic specificity.
\end{abstract}

\section{Key Words}

- thyroid carcinoma

- thyroid nodules

- Hashimoto's thyroiditis

- prognosis

- environment 


\section{Introduction}

Papillary thyroid carcinoma (PTC) is by far the most common histotype of thyroid cancer, accounting for about $80 \%$ of cases (Schlumberger 1998). Moreover, the rising incidence of thyroid malignancies, which has been recorded worldwide over the last three decades, is nearly totally attributable to increased diagnosis of the papillary histotype (Albores-Saavedra et al. 2007, Dal Maso et al. 2011, Davies \& Welch 2014). This has been demonstrated for all disease stages, but tumours confined to the thyroid were those showing the higher trend (Enewold et al. 2009) and currently represent the most common clinical scenario for PTC. Recently, dedicated clinico-pathological systems have been developed in order to quantify the risk of persistent/recurrent disease (Pacini et al. 2006, Pitoia et al. 2013), which represents the main prognostic goal. Nevertheless, long-term risk stratification of PTC is still suboptimal, as these systems failed to demonstrate adequate positive predictive value for the identification of recurring patients (Castagna et al. 2011). This particularly hampers the management of intrathyroidal PTCs, in which the 10-year risk of developing structural recurrences is extremely low, ranging from 0.5 to $5 \%$ (Ito et al. 2012). Therefore, management of this set of PTCs is currently controversial at different steps of the therapeutic process (extent of surgery, prophylactic central node dissection and radio-iodine treatment), also due to the absence of randomised controlled trials (McLeod et al. 2013). Hashimoto's thyroiditis (HT) is a complex disease characterised by diffuse lymphocytic infiltration determining progressive injury of the thyroid tissue, which leads to clinically evident hypothyroidism (Antonaci et al. 2009). Relationship between PTC and HT has been the focus of a wide body of research aiming to find out an association between the 2 conditions and to verify if co-existing HT affects clinico-pathological features and prognosis. Although conflicting results have been produced, a huge meta-analysis performed by Lee and coworkers (Lee et al. 2013) found that HT was significantly more frequent in patients with PTC, as compared with subjects carrying benign lesions as well as different tumour histotypes. Furthermore, authors also pointed out that co-occurrence of HT in PTCs not only was associated to protective features such as the absence of extrathyroidal extension and lymph node metastases, but, more importantly, was related to significantly lower recurrence rates. Nevertheless, the majority of performed studies were cross-sectional and therefore unable to provide proper evaluation of clinical outcome. More importantly, all of them included heterogeneous PTC cohorts, which were selected independently from disease extent. The aim of the present study was to define the prognostic value of histologically proven HT in intrathyroidal PTC.

\section{Patients and methods}

\section{Patients}

This was a multicentre study including 3 thyroid cancer referral units from Naples: University Federico II, University of Campania Luigi Vanvitelli and INT Pascale. In 2011, a common database was started recording clinico-pathological data of patients with differentiated thyroid cancer followed within the involved centres since 1997. Patients were registered retrospectively if operated before 2011 and prospectively thereafter. For the present study, inclusion criteria were (a) histological diagnosis of PTC (all variants were included) at pathological review; (b) intrathyroidal disease with inclusion of patients with primary tumour of any size but without extrathyroidal extension and showing no evidence of lymph nodes and distant sites involvement (subjects with post-surgery I-131 uptake outside thyroid bed were excluded) and (c) surgery and follow-up entirely performed at a single institution. Of the total 563 patients entered into the registry to date, 301 met the defined criteria. Data about clinical outcome were obtained by consulting the files and, if necessary, by recalling the patient himself. Follow-up was last updated in March 2017. The study was approved by the Ethics Committees of each included centre.

\section{Clinical management, outcome assessment and pathological evaluation}

Involved centres were tightly coordinated, with consistent treatment and follow-up procedures. The majority of intrathyroidal PTCs were treated by means of total thyroidectomy. Lymphadenectomy was not performed prophylactically, but only in case of clinical suspicious of lymph node involvement. Post-surgery RAI ablation was performed in accordance with dedicated guidelines from the referral societies (Silberstein et al. 2012). All patients undergoing RAI received low activity $(30-50 \mathrm{mCi})$. However, RAI treatment was not advised to patients with micro-PTC (pT1a according to the TNM system), unless they harboured aggressive histology (tall cell, diffuse sclerosing and solid variants)

Published by Bioscientifica Ltd. 
and/or somatic mutations with poor prognostic impact (such as the BRAFV600E). After thyroid ablation, patients were subjected to TSH suppressive therapy and underwent clinical (neck palpation), biochemical (thyroglobulin ( $\mathrm{Tg}$ ) $\mathrm{Tg}$ and $\mathrm{Tg}$ antibodies (AbTg) levels) and instrumental (neck ultrasonography (US)) follow-up every 6 months. Primary endpoint of the study was the rate of clinical remission lasting until final visit. Usually, the definition of clinical remission is consistent with the disease-free status, therefore including, as for the standard guidelines (Tuttle et al. 2010), patients with $\mathrm{Tg}$ (suppressed or stimulated) less than $1 \mathrm{ng} / \mathrm{mL}$, negative AbTg levels, absence of suspicious finding at neck US and no pathological evidence at any other study performed for clinically indicated reasons. However, the mentioned criteria did not fit our study as a remarkable portion of patients were expected to show what is currently defined as indeterminate response to therapy (Momesso \& Tuttle 2014). These include subjects undergoing surgery without RAI therapy, where Tg may show a progressive decline instead of a disappearance even when the disease is cured (Durante et al. 2012), and, more importantly, the study group of PTC with concomitant HT, where AbTg levels may remain positive for several years (Spencer 2011), likely due to immune memory rather than an actual disease persistence (Rubello et al. 1992, Dvorkin et al. 2013). Therefore, the definition of clinical remission in patients not treated with RAI and subjects with concomitant HT who showed detectable $\mathrm{Tg}$ and positive $\mathrm{AbTg}$, respectively, was based on the trend of marker concentrations and the detection of clinical or instrumental evidence of disease during a sufficiently long follow-up. Based on this assumption, assessment of clinical remission was partially affected by clinicians' subjective opinion. Therefore, in order to strengthen primary endpoint analysis, we considered auxiliary endpoint recurrence-free survival (RFS). In order to make the assessment as more objective as possible and also considering that biochemical recurrence corresponds to the presence of structural disease only in $20 \%$ of cases (Haugen et al. 2016), definition of RFS was based on the dates of structural recurrences, defined as any new morphological evidence of disease (detected at neck US or at any other instrumental examination, such as I-131 whole-body scan, radiography, computed tomography, 18-fluorodeoxyglucose positron emission tomography or biopsy) in subjects considered as cured after thyroid ablation. Pathological diagnosis of PTC was performed according to the World Health Organization criteria (DeLellis 2004). Co-existing HT was defined by the presence of diffuse/focal lymphoplasmacytic infiltrate, oxyphilic cells, lymphoid follicles with germinal centres and atrophic changes involving normal thyroid tissue, whereas isolated peri- and intra-tumoural lymphocytic infiltration was not considered as HT (McConahey 1972).

\section{Statistical analysis}

For all statistical analyses, SPSS, version 20.0 for Windows (SPSS Inc., Chicago, IL) was used. Group comparisons of categorical variables were performed by means of chisquare test and, in case of cell size less than 5, with Fisher's exact test (Lydersen et al. 2009). Cases where zeros caused problems with computation of the ORs and 95\% CIs were managed by adding 0.5 to all cells of the contingency table (Pagano \& Gauvreau 2000). Continuous variables, including age at diagnosis and primary tumour size, were analysed by means Mann-Whitney $U$ test for medians comparison. Survival analyses were performed according to the Kaplan-Meier method, and the log-rank test was used to test for differences between groups. Multivariate models of binary logistic regression analysis were applied for assessing if co-existing HT was an independent predictor of clinical remission. Accuracy of the non-HT status as prognostic marker was assessed according to the following formula: the positive predictive value (PPV) was true positive/(true positive +false positive) and the negative predictive value (NPV) was true negative/(false negative+true negative) (Galen 1982). All tests were two sided, and $P$ values of less than 0.05 were used for considering an association of statistical significance.

\section{Results}

\section{Study cohort}

Baseline clinico-pathological features and clinical outcome of the study cohort of intrathyroidal PTC were reported in Table 1. Mean follow-up was $75 \pm 59$ (s.D.) months (median 4.9 years). Out of 301 patients, 48 were males and 253 females. Median age at diagnosis was 38 years (range 15-71). Classic and follicular PTC was largely the most common variants accounting for 52.2 and $25.9 \%$ of cases, respectively. Nevertheless, the portion of aggressive/unfavourable variants was not negligible, including 28 cases (13 tall cell, 10 diffuse sclerosing and 5 solid PTCs) (9.3\%). The latter were the only patients to be considered at intermediate risk of recurrence/persistence according to the America Thyroid Association criteria (Pitoia et al. 2013), whereas the vast majority of the cohort $(90.7 \%)$ was composed of

Published by Bioscientifica Ltd 
Table 1 Baseline clinico-pathological characteristics and prognostic outcome of the overall cohort of intrathyroidal PTC.

\begin{tabular}{|c|c|}
\hline Parameter & Value \\
\hline Number of patients & 301 \\
\hline Age at diagnosis, median (range) & $38(15-71)$ \\
\hline Sex-ratio, males/females, number (\%) & $48 / 253(15.9 / 84.1)$ \\
\hline \multicolumn{2}{|l|}{ PTC variants, number $(\%)$} \\
\hline Classic & $157(52.2)$ \\
\hline Follicular & $78(25.9)$ \\
\hline Warthin-like & $24(8.0)$ \\
\hline Hürthle cells & $14(4.7)$ \\
\hline Tall cell & $13(4.3)$ \\
\hline Diffuse sclerosing & $10(3.3)$ \\
\hline Solid & $5(1.7)$ \\
\hline $\begin{array}{l}\text { Primary tumour size, median } \\
\text { (range) }(\mathrm{cm})\end{array}$ & $1.2(0.6-4)$ \\
\hline Micro-PTC, number (\%) & $117(38.9)$ \\
\hline Multifocality, number (\%) & $76(25.2)$ \\
\hline Co-existing $\mathrm{HT}$, number (\%) & $128(42.5)$ \\
\hline $\begin{array}{l}\text { No post-surgery RAI-treatment, } \\
\text { number }(\%)\end{array}$ & $68(22.6)$ \\
\hline $\begin{array}{l}\text { ATA risk group, low/intermediate/high, } \\
\text { number }(\%)\end{array}$ & $273 / 28 / 0(90.7 / 9.3 / 0.0)$ \\
\hline \multicolumn{2}{|l|}{ Clinical remission, number (\%) } \\
\hline Yes & $255(84.7)$ \\
\hline Disease-free & $236(78.4)$ \\
\hline Detectable Tg* & $0(0.0)$ \\
\hline AbTg positivity** & $19(6.3)$ \\
\hline No & $44(13.3)$ \\
\hline Structural recurrence & $32(10.6)$ \\
\hline Rising Tg & $12(4.0)$ \\
\hline Rising AbTg & $0(0.0)$ \\
\hline Uncertain*** & $2(0.7)$ \\
\hline
\end{tabular}

*Patients not subjected to post-surgery RAl showing stable/declining Tg levels with no other evidence of disease; **Patients with concomitant HT showing stable/declining AbTg levels with no other evidence of disease; $* * *$ Patients with non-specific imaging findings.

ATA, American Thyroid Association; HT, Hashimoto's thyroiditis; PTC, Papillary thyroid cancer; RAI, Radio-iodine. low-risk PTCs. Histologically proven HT was detected in 128 subjects (42.5\%). Among them, 118 (92.2\%) were AbTg positive before surgery, and the vast majority (99, 83.9\%) achieved negativisation during follow-up with a median disappearing time of $17 \pm 4.1$ months. Sixtyeight patients (22.6\%), all affected with micro-PTC, were not advised to RAI. Overall, 255 (84.7\%) patients were classified as being in clinical remission until final visit. Of them, the vast majority $(236,78.4 \%)$ met full criteria for the disease-free status, whereas the remaining portion $(19,16.1 \%)$ included patients with concomitant HT who showed stable/declining AbTg levels and no other evidence of disease after a mean follow-up of $63 \pm 42$ (s.D.) months (median 4.1 years). Among patients classified not in clinical remission, 32 (10.6\%) experienced documented structural recurrence, whereas 12 (4\%) showed rising Tg levels.

\section{Relationship between HT and clinico-pathological features at diagnosis}

Correlation between pathologically proven HT and baseline characteristics were reported in Table 2. A wide predominance of the female gender was observed in patients with HT, as compared with the control group $(P<0.001$, OR 4.51, 95\% CI 2.03-10.02). Primary tumour size was significantly smaller in case of concomitant HT (median 1 vs $1.3 \mathrm{~cm} ; P=0.008$ ). A strong correlation was found between HT and PTC histology $(P<0.001)$. In order to characterise such relationship, we performed a multiple testing analysis assessing the association between HT and different PTC histotypes (Table 3). We found that patients with HT were more likely affected

Table 2 Comparison of clinico-patological features between patients without and with co-existing HT in the study cohort of intrathyroidal PTC.

\begin{tabular}{|c|c|}
\hline Clinico-pathological features & PTC without co-existing HT \\
\hline Age at diagnosis, median (range) & $37(15-71)$ \\
\hline Gender, females, number $(\%)$ & $133 / 173(76.9)$ \\
\hline \multicolumn{2}{|l|}{ PTC variants, number $(\%)$} \\
\hline Classic & $96 / 173(55.5)$ \\
\hline Follicular & $36 / 173(20.8)$ \\
\hline Hürthle cells & 9/173 (5.2) \\
\hline Warthin-like & $6 / 173(3.5)$ \\
\hline Tall cell & $12 / 173(6.9)$ \\
\hline Solid & 9/173 (5.2) \\
\hline Diffuse sclerosing & $5 / 173(2.9)$ \\
\hline Primary tumour size, median (range) $(\mathrm{cm})$ & $1.3(0.7-4)$ \\
\hline Multifocality, number (\%) & $50 / 173(28.9)$ \\
\hline Post-surgery RAI-treatment, number (\%) & $143 / 173(82.7)$ \\
\hline
\end{tabular}

\begin{tabular}{c}
\hline PTC with co-existing H \\
\hline $39.5(17-64)$ \\
$120 / 128(93.8)$ \\
\\
$61 / 128(47.7)$ \\
$42 / 128(32.8)$ \\
$5 / 128(3.9)$ \\
$18 / 128(14.1)$ \\
$1 / 128(0.8)$ \\
$1 / 128(0.8)$ \\
$0 / 128(0)$ \\
$1(0.6-4)$ \\
$26 / 128(20.3)$ \\
$82 / 128(64.1)$
\end{tabular}

$\frac{\text { OR }(95 \% \mathrm{Cl})^{*}}{\mathrm{na}}$

$P$ value
0.195
$<\mathbf{0 . 0 0 1}$

*ORs with $95 \% \mathrm{Cl}$ were calculated using PTC without co-existing HT as reference.

$\mathrm{Cl}$, Confidence interval; HT, Hashimoto's thyroiditis; OR, Odds ratio; PTC, Papillary thyroid cancer; RAl, Radio-iodine. 
Table 3 Comparison of single PTC histotypes between patients without and with co-existing HT in the study cohort of intrathyroidal PTC.

\begin{tabular}{|c|c|c|}
\hline PTC variants & PTC without co-existing HT & PTC with co-existing HT \\
\hline Classic & $96 / 173(55.5)$ & $61 / 128(47.7)$ \\
\hline Follicular & $36 / 173(20.8)$ & $42 / 128(32.8)$ \\
\hline Hürthle cells & $9 / 173(5.2)$ & $5 / 128(3.9)$ \\
\hline Warthin-like & $6 / 173(3.5)$ & $18 / 128(14.1)$ \\
\hline Aggressive histology* & $26 / 173(15)$ & $2 / 128(1.6)$ \\
\hline
\end{tabular}

\begin{tabular}{l}
\multicolumn{1}{c}{ OR $(95 \% \mathrm{Cl})$} \\
\hline $0.73(0.46-1.15)$ \\
$1.85(1.1-3.12)$ \\
$0.74(0.24-2.26)$ \\
$4.58(1.76-11.9)$ \\
$0.08(0.02-0.38)$
\end{tabular}

\begin{tabular}{c} 
Unadjusted $\boldsymbol{P}$ value \\
\hline 0.178 \\
$\mathbf{0 . 0 1 8}$ \\
0.596 \\
$<\mathbf{0 . 0 0 1}$ \\
$<\mathbf{0 . 0 0 1}$
\end{tabular}

Adjusted $P$ value 0.356 0.054 0.596 $<0.001$ $<0.001$

*Owing to the low number of cases, the aggressive tall cell, diffuse sclerosing, and solid PTC variants were considered as a single group. $P$ values have been adjusted by means of the Holm-Bonferroni correction.

HT, Hashimoto's thyroiditis; PTC, Papillary thyroid cancer.

with the favourable Warthin-like $(P<0.001$, OR 4.58 , 95\% CI 1.76-11.9) and follicular $(P=0.018$, OR 1.85, 95\% CI 1.1-3.12) variants, whereas the risk of aggressive histology was significantly lower $(P<0.001$, OR 0.08 , 95\% CI 0.02-0.38), as compared with the control group. Nevertheless, significance of the association with the follicular variant was lost after correction by means of the Holm-Bonferroni method (Holm 1979). PTC with co-existing HT were less frequently subjected to postsurgery administration of I-131 ( $P=0.001$, OR $0.4,95 \% \mathrm{CI}$ $0.23-0.69)$, due to the reduced tumour volume.

\section{Relationship of HT with clinical remission and RFS}

Two patients with non-specific imaging findings were excluded from outcome analysis. Concomitant HT was

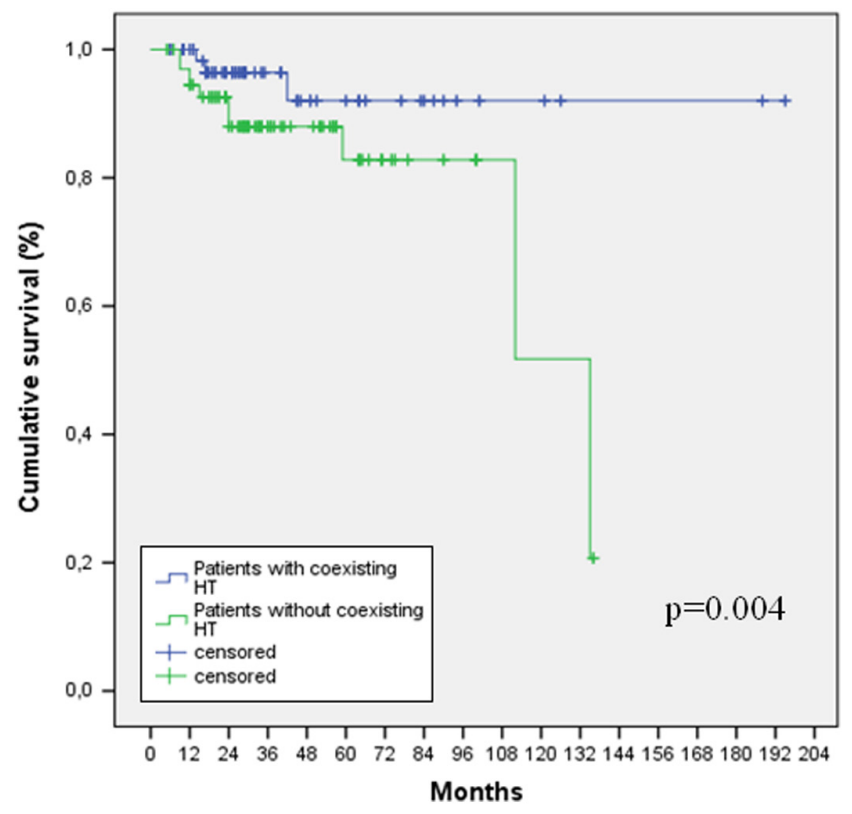

Figure 1

Kaplan-Meier analysis of recurrence-free survival according to co-existing HT. HT, Hashimoto's thyroiditis. associated to significantly higher likelihood of being in clinical remission during the whole follow-up time $(P<0.001$, OR 4, 95\% CI 1.78-8.94), and this represented our primary endpoint. Analysis of RFS, considered as auxiliary endpoint, included 295 subjects, as the time of recurrence was not unequivocally defined for 4 subjects. As reported in Fig. 1, PTCs with concomitant HT had significantly longer RFS, as compared with non-HT tumours $(P=0.004)$.

\section{Multivariate analysis of prognostic factors of clinical remission}

In order to test whether concomitant HT was an independent predictor of clinical remission, we built a multivariate adjusted model basing on clinicopathological features with possible prognostic impact on intrathyroidal PTC. Results were reported in Table 4. At univariate analysis, parameters other than HT showing relationship with clinical remission were age at diagnosis $\geq 45$ years $(P<0.001$, OR 9.42, 95\% CI 2.22-39.91), unfavourable PTC histology $(P=0.001$, OR $0.25,95 \%$ CI 0.11-0.6), microcarcinoma $(P<0.001$, OR 4.88, 95\% CI 1.99-11.95), multifocality $(P<0.001$, OR 0.27 , 95\% CI $0.14-0.52)$ and post-surgery RAI $(P<0.001$, OR $0.02,95 \%$ CI 0-0.48). Notably, RAI-treatment exerted a paradoxical deleterious prognostic impact being associated to lower likelihood of clinical remission. This result cannot be considered as demonstrating an actual prognostic effect for post-surgery RAI, but was rather due the fact that all patients not subjected to RAI were affected with microPTC and had therefore less aggressive disease. Owing to this consideration, post-surgery RAI was excluded from our multivariate model. Multivariate analysis confirmed co-existing HT as predictor of clinical remission $(P=0.006$, OR 3.28, 95\% CI 1.39-7.72), together with age at diagnosis $\geq 45$ years $(P=0.023$, OR $5.57,95 \%$ CI $1.26-24.53)$ and microcarcinoma $(P=0.010$, OR 3.40, 95\% CI 1.33-8.66).

Published by Bioscientifica Ltd 
Table 4 Multivariate analysis of prognostic factors of clinical remission in the cohort of intrathyroidal PTC.

\begin{tabular}{|c|c|c|c|c|c|c|}
\hline & \multicolumn{4}{|c|}{ Clinical remission, $\boldsymbol{N}(\%)$} & \multicolumn{2}{|c|}{ Adjusted multivariate model } \\
\hline & Yes & No & $\begin{array}{c}\text { Unadjusted OR } \\
\quad(95 \% \mathrm{Cl})\end{array}$ & $\begin{array}{c}\text { Unadjusted } \\
P \text { value }\end{array}$ & $\begin{array}{l}\text { Adjusted OR } \\
(95 \% \mathrm{Cl})^{* * *}\end{array}$ & Adjusted $P$ value \\
\hline \multicolumn{7}{|l|}{ Gender } \\
\hline Male & $38(79.2)$ & $10(20.8)$ & 1 (reference) & 0.192 & Not included & Not included \\
\hline Female & $217(86.5)$ & $34(13.5)$ & $1.67(0.76-3.68)$ & & & \\
\hline \multicolumn{7}{|l|}{ Age at diagnosis } \\
\hline$<45$ years & $176(80.7)$ & $42(19.3)$ & 1 (reference) & $<0.001$ & 1 (reference) & 0.023 \\
\hline$\geq 45$ years & 79 (97.5) & $2(2.5)$ & $9.42(2.22-39.91)$ & & $5.57(1.26-24.53)$ & \\
\hline \multicolumn{7}{|l|}{ Histology* } \\
\hline Favourable & $237(87.5)$ & $34(12.5)$ & 1 (reference) & 0.001 & 1 (reference) & 0.483 \\
\hline Unfavourable & $18(64.3)$ & $10(35.7)$ & $0.25(0.11-0.60)$ & & $0.69(0.25-1.91)$ & \\
\hline \multicolumn{7}{|l|}{ Tumour size** } \\
\hline Microcarcinoma & $111(94.9)$ & $6(5.1)$ & 4.88 (1.99-11.95) & $<0.001$ & $3.40(1.33-8.66)$ & 0.010 \\
\hline Macrocarcinoma & $144(79.1)$ & $38(20.9)$ & 1 (reference) & & 1 (reference) & \\
\hline \multicolumn{7}{|l|}{ Multifocality } \\
\hline Yes & $54(71.1)$ & $22(28.9)$ & $0.27(0.14-0.52)$ & $<0.001$ & $0.47(0.21-1.02)$ & 0.056 \\
\hline No & $119(90)$ & $22(10)$ & 1 (reference) & & 1 (reference) & \\
\hline \multicolumn{7}{|l|}{ Co-existing HT } \\
\hline Yes & $120(93.8)$ & $8(6.2)$ & $4.0(1.78-8.94)$ & $<0.001$ & $3.28(1.39-7.72)$ & 0.006 \\
\hline No & 135 (78.9) & $36(21.1)$ & 1 (reference) & & 1 (reference) & \\
\hline \multicolumn{7}{|l|}{ Post-surgery RAI } \\
\hline Yes & $179(80.3)$ & $44(19.7)$ & $0.02(0-0.48)$ & $<0.001$ & Not included & Not included \\
\hline No & $68(100)$ & $0(0)$ & 1 (reference) & & & \\
\hline
\end{tabular}

*Favourable PTC histology includes classic, follicular, Warthin-like, and Hurtle-cells variants; unfavourable histology includes tall cell, diffuse sclerosing, and solid variants; ${ }^{* *}$ Categorisation in micro- and macro- carcinoma was used for the analysis.

$\mathrm{Cl}$, Confidence interval; HT, Hashimoto's thyroiditis; N, number; OR, Odds ratio; RAl, Radio-iodine.

\section{Utility of the parameter HT in predicting poor clinical outcome}

Since the aim of PTC prognostication was to identify the subgroup of patients with unfavourable outcome, whereas HT revealed positive prognostic impact, we analysed the performance of the non-HT status as prognostic marker, specifically assessing its capability in identifying those subjects not maintaining clinical remission until the final visit. Despite limited by the low prevalence of the disease status, namely the absence of clinical remission, which was $14.7 \%$, the absence of HT showed limited specificity, namely $47.06 \%$ (95\% CI 40.80-53.38), and poor PPV, namely $21.05 \%$ (95\% CI 15.20-27.93). This suggests very limited utility of the parameter HT for assessing the risk of persistent/ recurrent disease.

\section{Discussion}

The development of an immune response has been reported in a significant portion of PTC, as demonstrated by the presence of inflammatory infiltration within or surrounding the tumour (French et al. 2010). Whether this immune microenvironment has an impact on PTC development and progression, and the underlying molecular mechanisms, is still a matter of discussion. Indeed, PTC-related immune network is extremely complex involving a myriad of cells (different lymphocyte subtypes, macrophages, mast cells and dendritic cells) and also a variety of soluble factors (cytokines, chemokines, angiogenic and lymphangiogenic factors) (Galdiero et al. 2016). To date, available data pointed out a relationship between intra- and/or peri- tumoural inflammatory activation, mainly assessed by the presence of infiltrating lymphocytes, and favourable outcome, namely a reduced risk of disease recurrence (Matsubayashi et al. 1995, Gupta et al. 2001). Owing to these considerations, possible impact of co-existing HT on PTC outcome is an intriguing issue. Indeed, HT relies on an aberrant immune reaction specifically directed against the thyroid, which involves several immune cells as well as soluble mediators and leads to wide inflammatory infiltration of the gland (Weetman 2013). Actually, it is still controversial whether the previously mentioned tumour infiltrating lymphocytes represents an independent entity having own prognostic significance, as reported by French and coworkers (French et al. 2010), or has to be considered as inserted in the context of concomitant HT, as suggested by Kebebew and coworkers (Kebebew et al. 2001). Among a series of

Published by Bioscientifica Ltd 
prognostic studies having sufficiently long follow-up, the vast majority reported more favourable outcome for PTC when co-existing HT was detected (Matsubayashi et al. 1995, Loh et al. 1999, Kashima et al. 1998, Kebebew et al. 2001, Kim et al. 2009, Ahn et al. 2011, Huang et al. 2011, Jeong et al. 2012, Dvorkin et al. 2013). Nevertheless, HT revealed as an independent prognostic factor only in some of them (Kashima et al. 1998,Loh et al. 1999, Dvorkin et al. 2013). However, prognostic value of HT in PTC is still considered controversial because of the following issues: (a) some of the mentioned papers involved mainly but not exclusively the papillary histotype (Loh et al. 1999, Dvorkin et al. 2013); (b) criteria for HT definition were heterogeneous: some authors included not only patients with full histopathological features of HT (as previously described McConahey 1972), but also subjects with local tumour lymphocytic infiltration alone (Loh et al. 1999, Kim et al. 2009), whereas some others considered both clinics and histology for HT diagnosis (Dvorkin et al. 2013); (c) all studies were focused on heterogeneous cohorts involving all grades of disease extent. In our opinion, the latter was a crucial limitation as the interaction between HT-related microenvironment and PTC cells occurs solely within the thyroid, so the actual effect of HT on tumour evolution could be lost or at least diluted when the disease goes behind the gland. Furthermore, it is conceivable that PTC with HT is diagnosed at an earlier stage because of the more accurate ultrasonographic screening, and this may hamper the proper evaluation of the correlation between HT and tumour behaviour. To the best of our knowledge, this is the first study reporting significant data about the prognostic impact of concomitant HT on a large PTC cohort with intrathyroidal disease. Previously, Matsubayashi and coworkers (Matsubayashi et al. 1995) have tried to determine the prognostic role of local tumour lymphocytic infiltration according to the extent of PTC at the time of initial treatment, but sample size was too low for obtaining significant results. Another crucial characteristic of our study was that HT definition was based solely on histology. Particularly, only patients showing the typical histopathological changes in the area of normal thyroid parenchyma were included in the co-existing HT group, whereas those with localised tumour lymphocytic infiltration alone were considered as controls. In the present study, co-existing HT was detected in $42.5 \%$ of PTC, a prevalence higher than that reported in any other series (ranging from 0.5 to 38\%) (Lee et al. 2013). This could be explained by the association between HT and less advanced disease stage, which has been reported in many studies (Loh et al. 1999, French et al. 2010, Huang et al. 2011). Another possible reason is that the majority of patients lived in a highly polluted area such as Naples and the neighbour countries (Grumetto etal. 2015), and overexposure to thyroid-disrupting chemicals may facilitate the development of the autoimmune process in subjects predisposed to HT (Colucci et al. 2015). Analysis of baseline features revealed that primary tumour size was smaller in case of concomitant HT. This is an interesting finding as the majority of previous studies including all PTC stages failed to demonstrate this association (Lee et al. 2013). It may be hypothesised that analysing only intrathyroidal disease actually reveals the counteracting action of HT against local tumour growth. Even more interestingly, we also demonstrated a relationship between HT and PTC histology. Particularly, patients with concomitant HT showed significantly lower risk of aggressive variants. Furthermore, a strong relationship was found with the favourable Warthin-like and, even if statistical significance was lost after correction with the Holm-Bonferroni method, follicular PTC. However, assessment of relationship with the follicular variant was also hampered by the fact that histological definition pre-dated the recent reclassification of encapsulated tumours such as a 'noncancerous or premalignant neoplasm', named 'noninvasive follicular thyroid tumour with papillary-like nuclear features' (NIFTP). With the exception of the strong association with the Warthin-like variant, which has been long established (Baloch \& LiVolsi 2000), no evidence about the relationship between HT and PTC histology had been reported before. Analysis of clinical outcome revealed that concomitant HT was associated to higher likelihood of achieving and maintaining clinical remission until final visit. Furthermore, PTCs with HT showed higher RFS at survival analysis. Prognostic value of concomitant HT was further assessed by adjusting for parameters showing significant relationship with clinical remission at univariate analysis. These include age at diagnosis, primary tumour size, multifocality, histology and postsurgery RAI. Although deleterious effect related to higher tumour size and the presence of multifocality as well as aggressive histology was expected and already reported (Ito et al. 2012, Ghossein et al. 2014), protective role of older age ( $\geq 45$ years) and deleterious effect of post-surgery RAI appeared controversial. Actually, the former was consistent with the recent Ito study (Ito et al. 2014) showing that patients with non-operated low-risk PTC have lower risk of disease progression when age is $\geq 40$ years, whereas the latter can be interpreted as

Published by Bioscientifica Ltd 
exclusively related to the strict correlation with tumour size and was therefore excluded from the multivariate model. After adjustment, co-existing HT retained its protective effect, and this represents the main finding of our study. Recently, the Thyroid Cancer Genome Atlas has identified two main PTC groups with different genetics, pathology and clinical behaviour, namely RAS- and BRAFmutated tumours (Agrawal et al. 2014). Considering our findings, it may be speculated that HT-related PTC represents an independent clinical entity belonging to the RAS-mutated group, which predominantly includes the follicular variant histology and has better outcome, whereas the BRAF-mutated tumours involve the vast majority of aggressive variants and show more aggressive behaviour. However, this remains just a speculation and further studies, also including molecular characterisation, are needed to confirm this thesis. To date, HT is not included within the subset of variables to be considered for estimating clinical outcome of PTC (Haugen et al. 2016). Results from this study not only empower the thesis of a prognostic value of HT in PTC, but also provide insights about the possible application into clinical practice. Indeed, based on the current prognostic criteria (Pacini et al. 2006, Pitoia et al. 2013), our study population of intrathyroidal PTC was mainly composed of tumours with low-risk of recurrent/persistent disease, with the only exception of those few patients showing aggressive histology, who had to be collocated in the intermediaterisk class. Therefore, our findings can be considered as substantially referring to the setting of low-risk PTC, whereas further studies are needed to assess prognostic value of HT in other risk categories. Since the capability of identifying PTC with less favourable outcome represents the main limitation of current PTC prognostication, especially in patients with early disease, we analysed the accuracy of the non-HT status, considered as a negative prognosticator. We found that the absence of HT had low specificity and PPV for the identification of subjects not showing remission until the final visit, thus demonstrating the limited utility of the HT parameter for the actual improvement of PTC prognostication. In conclusion, we demonstrated that concomitant HT, as detected through pathological examination, is an independent prognostic factor in intrathyroidal PTC, but cannot improve prognostic specificity.

\section{Declaration of interest}

The authors declare that there is no conflict of interest that could be perceived as prejudicing the impartiality of the research reported.

\section{Funding}

This work was supported by the Umberto Veronesi Foundation, which granted the lead author Vincenzo Marotta with a postdoctoral Fellowship award for 2017.

\section{Acknowledgements}

The authors acknowledge the Umberto Veronesi Foundation for the postdoctoral Fellowship award 2017 provided to Vincenzo Marotta.

\section{References}

Agrawal AR, Aksoy BA, Ally A, Arachchi H, Asa SL, Auman JT, Balasundaram M, Balu S, Baylin SB, Behera M, et al. 2014 Integrated genomic characterization of papillary thyroid carcinoma. Cell 159 676-690. (doi:10.1016/j.cell.2014.09.050)

Ahn D, Heo SJ, Park JH, Kim JH, Sohn JH, Park JY, Park SK \& Park J 2011 Clinical relationship between Hashimoto's thyroiditis and papillary thyroid cancer. Acta Oncologica 50 1228-1234. (doi:10.3109 /0284186X.2011.602109)

Albores-Saavedra J, Henson DE, Glazer E \& Schwartz AM 2007 Changing patterns in the incidence and survival of thyroid cancer with follicular phenotype - papillary, follicular, and anaplastic: a morphological and epidemiological study. Endocrine Pathology 18 1-7. (doi:10.1007/s12022-007-0002-z)

Antonaci A, Consorti F, Mardente S \& Giovannone G 2009 Clinical and biological relationship between chronic lymphocytic thyroiditis and papillary thyroid carcinoma. Oncology Research 17 495-503. (doi:10.3727 /096504009789735431)

Baloch ZW \& LiVolsi VA 2000 Warthin-like papillary carcinoma of the thyroid. Archives of Pathology and Laboratory Medicine Online 124 1192-1195.

Castagna MG, Maino F, Cipri C, Belardini V, Theodoropoulou A, Cevenini G \& Pacini F 2011 Delayed risk stratification, to include the response to initial treatment (surgery and radioiodine ablation), has better outcome predictivity in differentiated thyroid cancer patients. European Journal of Endocrinology 165 441-446. (doi:10.1530/EJE-11-0466)

Colucci R, Lotti F, Arunachalam M, Lotti T, Dragoni F, Benvenga S \& Moretti S 2015 Correlation of serum thyroid hormones autoantibodies with self-reported exposure to thyroid disruptors in a group of nonsegmental vitiligo patients. Archives of Environmental Contamination and Toxicology 69 181-190. (doi:10.1007/s00244-015-0138-7)

Dal Maso L, Lise M, Zambon P, Falcini F, Crocetti E, Serraino D, Cirilli C, Zanetti R, Vercelli M, Ferretti S, et al. 2011 Incidence of thyroid cancer in Italy, 1991-2005: time trends and age-period-cohort effects. Annals of Oncology 22 957-963. (doi:10.1093/annonc/mdq467)

Davies L \& Welch HG 2014 Current thyroid cancer trends in the United States. JAMA Otolaryngology - Head and Neck Surgery 140 317-322.

DeLellis RA, Lloyd RV, Heitz PU \& Eng C 2004 Pathology and genetics of tumours of endocrine organs. In WHO/IARC Classification of Tumours, 3rd edn. Lyon, France: IARC Press.

Durante C, Montesano T, Attard M, Torlontano M, Monzani F, Costante G, Meringolo D, Ferdeghini M, Tumino S, Lamartina L, et al. 2012 Longterm surveillance of papillary thyroid cancer patients who do not undergo postoperative radioiodine remnant ablation: is there a role for serum thyroglobulin measurement? Journal of Clinical Endocrinology and Metabolism 97 2748-2753. (doi:10.1210/jc.2012-1123)

Dvorkin S, Robenshtok E, Hirsch D, Strenov Y, Shimon I \& Benbassat CA 2013 Differentiated thyroid cancer is associated with less aggressive disease and better outcome in patients with coexisting Hashimotos thyroiditis. Journal of Clinical Endocrinology and Metabolism 98 2409-2414. (doi:10.1210/jc.2013-1309)

Enewold L, Zhu K, Ron E, Marrogi AJ, Stojadinovic A, Peoples GE \& Devesa SS 2009 Rising thyroid cancer incidence in the United States

Published by Bioscientifica Ltc. http://erc.endocrinology-journals.org

DOI: 10.1530/ERC-17-0085
(C) 2017 Society for Endocrinology Printed in Great Britain 
by demographic and tumor characteristics, 1980-2005. Cancer Epidemiology, Biomarkers and Prevention 18 784-791. (doi:10.1158/1055-9965.EPI-08-0960)

French JD, Weber ZJ, Fretwell DL, Said S, Klopper JP \& Haugen BR 2010 Tumor-associated lymphocytes and increased FoxP3+ regulatory $\mathrm{T}$ cell frequency correlate with more aggressive papillary thyroid cancer. Journal of Clinical Endocrinology and Metabolism 95 2325-2333. (doi:10.1210/jc.2009-2564)

Galdiero MR, Varricchi G \& Marone G 2016 The immune network in thyroid cancer. Oncoimmunology 5 e1168556. (doi:10.1080/21624 02X.2016.1168556)

Galen RS 1982 Application of the predictive value model in the analysis of test effectiveness. Clinics in Laboratory Medicine 2 685-699.

Ghossein R, Ganly I, Biagini A, Robenshtok E, Rivera M \& Tuttle RM 2014 Prognostic factors in papillary microcarcinoma with emphasis on histologic subtyping: a clinicopathologic study of 148 cases. Thyroid 24 245-253. (doi:10.1089/thy.2012.0645)

Grumetto L, Ortosecco G, Russo G, Guida M, Ferranti P, Nasi A \& Barbato F 2015 Dioxin-like PCB levels in maternal and umbilical cord sera of people living near dump sites in southern Italy: a pilot study of biomonitoring. Environmental Monitoring and Assessment 187 88. (doi:10.1007/s10661-015-4267-2)

Gupta S, Patel A, Folstad A, Fenton C, Dinauer CA, Tuttle RM, Conran R \& Francis GL 2001 Infiltration of differentiated thyroid carcinoma by proliferating lymphocytes is associated with improved disease-free survival for children and young adults. Journal of Clinical Endocrinology and Metabolism 86 1346-1354. (doi:10.1210/jc.86.3.1346)

Haugen BR, Alexander EK, Bible KC, Doherty GM, Mandel SJ, Nikiforov YE, Pacini F, Randolph GW, Sawka AM, Schlumberger M, et al. 2016 American thyroid association management guidelines for adult patients with thyroid nodules and differentiated thyroid cancer: the American thyroid association guidelines task force on thyroid nodules and differentiated thyroid cancer. Thyroid 26 1-133. (doi:10.1089/thy.2015.0020)

Holm S 1979 A simple sequential rejective method procedure. Scandinavian Journal of Statistics 6 65-70.

Huang BY, Hseuh C, Chao TC, Lin KJ \& Lin JD 2011 Well-differentiated thyroid carcinoma with concomitant Hashimoto's thyroiditis present with less aggressive clinical stage and low recurrence. Endocrine Pathology 22 144-149. (doi:10.1007/s12022-011-9164-9)

Ito Y, Kudo T, Kihara M, Takamura Y, Kobayashi K, Miya A \& Miyauchi A 2012 Prognosis of low-risk papillary thyroid carcinoma patients: its relationship with the size of primary tumors. Endocrine Journal $\mathbf{5 9}$ 119-125. (doi:10.1507/endocri.EJ11-0288)

Ito Y, Miyauchi A, Kihara M, Higashiyama T, Kobayashi K \& Miya A 2014 Patient age is significantly related to the progression of papillary microcarcinoma of the thyroid under observation. Thyroid 24 27-34. (doi:10.1089/thy.2013.0367)

Jeong JS, Kim HK, Lee CR, Park S, Park JH, Kang SW, Jeong JJ, Nam KH, Chung WY \& Park CS 2012 Coexistence of chronic lymphocytic thyroiditis with papillary thyroid carcinoma: clinical manifestation and prognostic outcome. Journal of Korean Medical Science $\mathbf{2 7}$ 883-889. (doi:10.3346/jkms.2012.27.8.883)

Kashima K, Yokoyama S, Noguchi S, Murakami N, Yamashita H, Watanabe S, Uchino S, Toda M, Sasaki A, Daa T, et al. 1998 Chronic thyroiditis as a favorable prognostic factor in papillary thyroid carcinoma. Thyroid 8 197-202. (doi:10.1089/thy.1998.8.197)

Kebebew E, Treseler PA, Ituarte PH \& Clark OH 2001 Coexisting chronic lymphocytic thyroiditis and papillary thyroid cancer revisited. World Journal of Surgery 25 632-637. (doi:10.1007/s002680020165)

Kim EY, Kim WG, Kim WB, Kim TY, Kim JM, Ryu JS, Hong SJ, Gong G \& Shong YK 2009 Coexistence of chronic lymphocytic thyroiditis is associated with lower recurrence rates in patients with papillary thyroid carcinoma. Clinical Endocrinology 71 581-586. (doi:10.1111/j.1365-2265.2009.03537.x)

Lee JH, Kim Y, Choi JW \& Kim YS 2013 The association between papillary thyroid carcinoma and histologically proven Hashimoto's thyroiditis: a meta-analysis. European Journal of Endocrinology 168 343-349. (doi:10.1530/EJE-12-0903)

Loh KC, Greenspan FS, Dong F, Miller TR \& Yeo PP 1999 Influence of lymphocytic thyroiditis on the prognostic outcome of patients with papillary thyroid carcinoma. Journal of Clinical Endocrinology and Metabolism 84 458-463. (doi:10.1210/jcem.84.2.5443)

Lydersen S, Fagerland MW \& Laake P 2009 Recommended tests for association in $2 \times 2$ tables. Statistics in Medicine 28 1159-1175. (doi:10.1002/sim.3531)

Matsubayashi S, Kawai K, Matsumoto Y, Mukuta T, Morita T, Hirai K, Matsuzuka F, Kakudoh K, Kuma K \& Tamai H 1995 The correlation between papillary thyroid carcinoma and lymphocytic infiltration in the thyroid gland. Journal of Clinical Endocrinology and Metabolism $\mathbf{8 0}$ 3421-3424. (doi:10.1210/jc.80.12.3421)

McConahey WM 1972 Hashimoto's thyroiditis. Medical Clinics of North America 56 885-896. (doi:10.1016/S0025-7125(16)32354-9)

McLeod DS, Sawka AM \& Cooper DS 2013 Controversies in primary treatment of low-risk papillary thyroid cancer. Lancet $\mathbf{3 8 1}$ 1046-1057. (doi:10.1016/S0140-6736(12)62205-3)

Momesso DP \& Tuttle RM 2014 Update on differentiated thyroid cancer staging. Endocrinology Metabolism Clinics of North America 43 401-421. (doi:10.1016/j.ecl.2014.02.010)

Pacini F, Schlumberger M, Dralle H, Elisei R, Smit JW \& Wiersinga W 2006 European consensus for the management of patients with differentiated thyroid carcinoma of the follicular epithelium. European Journal of Endocrinology 154 787-803. (doi:10.1530/eje.1.02158)

Pagano M \& Gauvreau K 2000 Principles of Biostatistics. 2nd ed. Belmont, CA, USA: Duxbury Press.

Pitoia F, Bueno F, Urciuoli C, Abelleira E, Cross G \& Tuttle RM 2013 Outcomes of patients with differentiated thyroid cancer riskstratified according to the American thyroid association and Latin American thyroid society risk of recurrence classification systems. Thyroid 23 1401-1407. (doi:10.1089/thy.2013.0011)

Rubello D, Casara D, Girelli ME, Piccolo M \& Busnardo B 1992 Clinical meaning of circulating antithyroglobulin antibodies in differentiated thyroid cancer: a prospective study. Journal of Nuclear Medicine $\mathbf{3 3}$ $1478-1480$

Schlumberger MJ 1998 Papillary and follicular thyroid carcinoma. New England Journal of Medicine 338 297-306. (doi:10.1056/ NEJM199801293380506)

Silberstein EB, Alavi A, Balon HR, Clarke SE, Divgi C, Gelfand MJ, Goldsmith SJ, Jadvar H, Marcus CS, Martin WH, et al. 2012 The SNMMI practice guideline for therapy of thyroid disease with 131I 3.0. Journal of Nuclear Medicine 53 1633-1651. (doi:10.2967/jnumed.112.105148)

Spencer CA 2011 Clinical review: clinical utility of thyroglobulin antibody $(\mathrm{TgAb})$ measurements for patients with differentiated thyroid cancers (DTC). Journal of Clinical Endocrinology and Metabolism 96 3615-3627. (doi:10.1210/jc.2011-1740)

Tuttle RM, Tala H, Shah J, Leboeuf R, Ghossein R, Gonen M, Brokhin M, Omry G, Fagin JA \& Shaha A 2010 Estimating risk of recurrence in differentiated thyroid cancer after total thyroidectomy and radioactive iodine remnant ablation: using response to therapy variables to modify the initial risk estimates predicted by the new American Thyroid Association staging system. Thyroid 20 1341-1349. (doi:10.1089/thy.2010.0178)

Weetman AP 2013 The immunopathogenesis of chronic autoimmune thyroiditis one century after hashimoto. European Thyroid Journal 1 243-250.

Received in final form 5 July 2017

Accepted 10 July 2017

Accepted Preprint published online 10 July 2017 http://erc.endocrinology-journals.org

DOI: 10.1530/ERC-17-0085
(C) 2017 Society for Endocrinology Printed in Great Britain 